\title{
Crystal structures of two thiacalix[4]arene derivatives anchoring four thiadiazole groups
}

\author{
BANG-TUN ZHAO ${ }^{\mathrm{a}, \mathrm{b}, *}$, ZHEN ZHOU $^{\mathrm{b}}$ and ZHEN-NING YAN \\ ${ }^{a}$ Department of Chemistry, Luoyang Normal University, Luoyang 471022, PR China \\ ${ }^{b}$ Department of Chemistry, Zhengzhou University, Zhengzhou 450001, PR China \\ e-mail: zbt@lynu.edu.cn
}

MS received 6 August 2008; revised 31 May 2009; accepted 12 June 2009

\begin{abstract}
The crystal structures of two thiacalixarene derivatives anchoring thiadiazole functional groups at lower rim, $\mathrm{C}_{60} \mathrm{H}_{72} \mathrm{O}_{4} \mathrm{~S}_{12} \mathrm{~N}_{8}(1), \mathrm{C}_{64} \mathrm{H}_{80} \mathrm{O}_{4} \mathrm{~S}_{12} \mathrm{~N}_{8}$ (2), have been determined by single crystal X-ray diffraction. The thiacalix[4]arene framework in both 1 and 2 adopts the 1,3-alternate conformation. Compound 1 forms a 1-D chain by weak hydrogen bonding $(\mathrm{C}-\mathrm{H} \cdots \mathrm{N})$ interactions between two thiadiazole groups in two different molecules. The chains are further connected to form a 2-D network through sulfur-sulfur $(\mathrm{S} \cdots \mathrm{S})$ interactions. The lattice water molecules which exist as dimers by forming hydrogen bonds $(\mathrm{O}-\mathrm{H} \cdots \mathrm{O})$ promote a $3-\mathrm{D}$ supramolecular structure through weak hydrogen bonding $(\mathrm{O}-\mathrm{H} \cdots \mathrm{S})$ interactions between the lattice water dimers and the 2-D networks. On the other hand, compound 2, based on dimer which is formed by weak hydrogen bonding $(\mathrm{C}-\mathrm{H} \cdots \mathrm{S})$ interactions, is extended to a $1-\mathrm{D}$ chain through sulfur-sulfur (S...S) interactions. The dimers of lattice methanol molecules linked by hydrogen bonds $(\mathrm{O}-\mathrm{H} \cdots \mathrm{O})$ act as bridges to link the 1-D chains into a 2-D network through weak hydrogen bonding $(\mathrm{C}-\mathrm{H} \cdots \mathrm{N})$ interactions.
\end{abstract}

Keywords. Thiacalixarene; thiadiazole; crystal structure; weak interaction.

\section{Introduction}

As novel members of the well-known calixarene family ${ }^{1-4}$ thiacalixarenes since their discovery in 1997; have attracted considerable interest as an alternative to 'classic' calixarenes by providing sites for functionallization not only on the aromatic rings but also on the bridging sulfur atoms. ${ }^{5}$ By virtue of electron-rich sulfur bridges, thiacalixarenes possess additional coordination sites and flexible cavity dimensions as well as high affinity to transition metal ions compared with analogous calixarenes, and thus are considered to be superior to the classical calixarenes. ${ }^{6,7}$ The conformational preferences of thiacalix[4]arene derivatives in the solid state are controlled by the groups attached to the thiacalixarene framework, presence of guest molecule(s), solvent of crystallization and other factors. ${ }^{8,9}$ In a previous paper, we have synthesized novel thiacalixarene derivatives bearing thiadiazole functional groups at lower rims. ${ }^{10}$ In the present study, we report the crystal structures of two

*For correspondence thiacalix[4]arene derivatives which form supramolecular structures through intermolecular interactions.

\section{Experimental}

\subsection{Synthesis of $\mathrm{C}_{60} \mathrm{H}_{72} \mathrm{O}_{4} \mathrm{~S}_{12} \mathrm{~N}_{8}(\mathbf{1}), \mathrm{C}_{64} \mathrm{H}_{80} \mathrm{O}_{4} \mathrm{~S}_{12} \mathrm{~N}_{8}$ (2)}

Compounds 1 and 2 (figure 1) were synthesized as white powder as per the reported procedure. ${ }^{10} \mathrm{~A}$ sample of 1 or $2 \sim 30 \mathrm{mg}$ was dissolved in $30 \mathrm{~mL}$ chloroform. Several drops of methanol were added and the solution was allowed to evaporate for slowly a week at room temperature to obtain single crystals suitable for X-ray diffraction analysis.

\section{$2.2 X$-ray structure determination}

$\mathrm{X}$-ray single-crystal data collections for $\mathbf{1}$ and $\mathbf{2}$ were performed with a Bruker SMART APEX II CCD diffractometer equipped with a graphite monochromated Mo-K $\alpha$ radiation $(\lambda=0.71073 \AA)$ by using $\phi-\omega$ scan technique at room temperature. The structures were solved by direct methods and refined 
by the full-matrix least-squares refinement on $F^{2}$ using with SHELXL-97 package. ${ }^{11-13}$ Intensity data were corrected for empirical absorption. All the nonhydrogen atoms were refined anisotropically. Hydrogen atoms of water molecules were located by Fourier map, and then refined by riding mode. The other hydrogen atoms were assigned with common isotropic displacement factors and included in the final refinement by use of geometrical restrains. A summary of the crystallographic data are given in table 1 . The $R$ factors for both the structures are quite high presumably due to poor quality of the crystals. Selected bond distances and angles are given in tables 2 and 3 , respectively. The geometrical parameters of hydrogen bonding and other intermolecular interactions are listed in table 4.

\section{Results and discussion}

\subsection{Structure of $\mathrm{C}_{60} \mathrm{H}_{72} \mathrm{O}_{4} \mathrm{~S}_{12} \mathrm{~N}_{8}(1) \cdot \mathrm{CHCl}_{3} \cdot \mathrm{H}_{2} \mathrm{O}$}

The thiacalix[4]arene framework adopts the 1,3alternate conformation. As seen from figure 2, the asymmetric unit consists of one thiacalix[4]arene molecule and one chloroform and one water molecules. The dihedral angle between the two thiadiazole rings on the same plane defined by the four sulfur atoms of thiacalix[4]arene framework is $77.3^{\circ}, 10.9^{\circ}$, respectively. For every two opposite benzene rings of thiacalix[4]arene framework, the dihedral angle and centroid distance are $48.48(1)^{\circ}$, 6.43(3) $\AA, 54.89(2)^{\circ}, 6.53(3) \AA$, respectively. The four sulfur atoms on thiacalix[4]arene skeleton lie in the same plane with a mean deviation of $0.08 \AA$. The distance between two opposite sulfur atoms as well as oxygen atoms are 7.93(3) $\AA, 7 \cdot 87(3) \AA$,

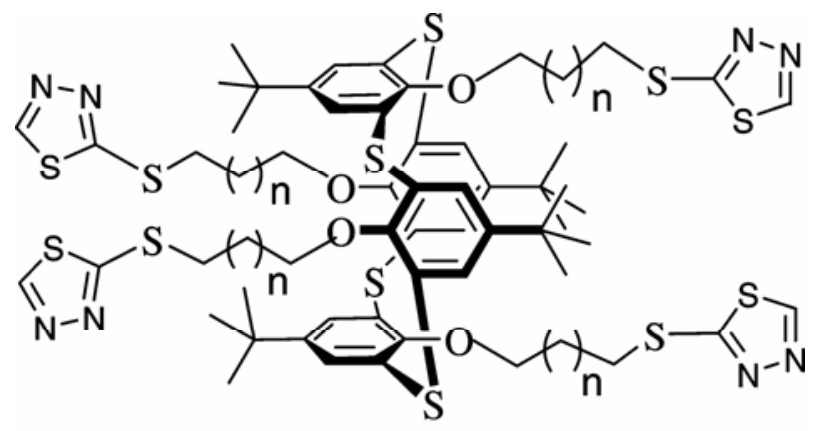

$$
1, n=1 \quad 2, n=2
$$

Figure 1. The structures of compounds $\mathbf{1}$ and $\mathbf{2}$.
4.25(7) $\AA, 3.98(2) \AA$, respectively. In the crystal structure, the molecules are linked to form 1-D chain structure by weak hydrogen bonding $\left(\mathrm{C}_{50}-\right.$ $\mathrm{H}_{50} \cdots \mathrm{N}_{4}=3.25 \AA$ and $\mathrm{C}_{60}-\mathrm{H}_{60} \cdots \mathrm{N}_{5}=3.40 \AA$ ) interactions $^{14}$ between thiadiazole nitrogen atoms and hydrogen atoms of methyl groups attached to another thiadiazole ring (figure 3). The adjacent 1-D chains are further connected to form a 2-D network through weak sulfur-sulfur $\left(\mathrm{S}_{6} \cdots \mathrm{S}_{11}=3.59 \AA\right)^{15}$ interactions between sulfur atoms in the thiadiazole rings and 2-positional mercapto sulfur atoms (as seen from supplementary material figure $\mathrm{S} 1$ ). The lattice water molecules, which exist as dimers through hydrogen bondings $\left(\mathrm{O}_{5}-\mathrm{H}_{2} \cdots \mathrm{O}_{5}=\right.$ $2.28 \AA$ ), promote the formation of a 3-D network by weak hydrogen bonding $\left(\mathrm{O}_{5}-\mathrm{H}_{1} \cdots \mathrm{S}_{12}=3.78 \AA\right)^{16,17}$ interactions between the lattice water dimers and 2D networks (see figure S2 and figure S3 of supplementary material). The lattice chloroform molecules do not play any roles in the supramolecular structures.

\subsection{Structure of $\mathrm{C}_{64} \mathrm{H}_{80} \mathrm{~N}_{8} \mathrm{O}_{6} \mathrm{~S}_{12}$ (2) $2 \mathrm{CH}_{3} \mathrm{OH}$}

As shown in figure 4 , the asymmetric unit of the compound $\mathrm{C}_{64} \mathrm{H}_{80} \mathrm{~N}_{8} \mathrm{O}_{6} \mathrm{~S}_{12}$ (2) $\cdot 2 \mathrm{CH}_{3} \mathrm{OH}$ contains one thiacalix[4]arene molecule and two methanol molecules. The dihedral angle between the two thiadiazole rings on the same side is $82.0^{\circ}, 25.0^{\circ}$, respectively. For every two opposite benzene rings of thiacalix[4]arene framework, the dihedral angle and centroid distance are $43 \cdot 74(2)^{\circ}, 6 \cdot 28(7) \AA, 50 \cdot 26(2)^{\circ}$, 6.44(6) $\AA$, respectively. The four sulfur atoms on calix[4]arene skeleton lie in the same plane with the mean deviation of $0.19 \AA$. The distance between the two opposite sulfur atoms as well as oxygen atoms are 7.90(3) $\AA, 7 \cdot 80(2) \AA, 4 \cdot 18(7) \AA, 4 \cdot 14(6) \AA$, respectively. Two molecules of $\mathbf{2}$ are connected to form a dimer through two weak hydrogen bonding $\left(\mathrm{C}_{49}-\mathrm{H}_{49} \cdots \mathrm{S}_{2}=3.70 \AA\right)^{18}$ interactions involving the hydrogen atoms from alkyl chains and the sulfur atoms of thiacalix[4]arene framework. These dimers are assembled into 1-D chain structure by sulfursulfur $(\mathrm{S} \cdots \mathrm{S}=3.65 \AA)^{14}$ interactions between the sulfur atoms from adjacent mercaptothiadiazole groups (see figure S4 of supplementary material). The lattice methanol molecules exist in dimers by hydrogen bondings $\left(\mathrm{O}_{5}-\mathrm{H}_{5} \cdots \mathrm{O}_{6}=3.05 \AA\right.$ and $\mathrm{O}_{6}$ $\mathrm{H}_{6} \cdots \mathrm{O}_{5}=3.05 \AA$ ). Subsequently, a 2-D network is generated through weak hydrogen bonding $\left(\mathrm{C}_{65}-\mathrm{H}_{65} \ldots \mathrm{N}_{2}=3.35 \AA\right.$ and $\left.\mathrm{C}_{46}-\mathrm{H}_{46} \cdots \mathrm{O}_{6}=3.42 \AA\right)$ 
Table 1. Crystal data and structure refinement parameters for $\mathbf{1}$ and 2.

\begin{tabular}{lll}
\hline Compound & \multicolumn{1}{c}{$\mathbf{1}$} & \multicolumn{2}{c}{$\mathbf{2}$} \\
\hline Formula & $\mathrm{C}_{61} \mathrm{H}_{75} \mathrm{~N}_{8} \mathrm{O}_{4} \mathrm{~S}_{12}(\mathbf{1}) \cdot \mathrm{H}_{2} \mathrm{O} \cdot \mathrm{CHCl}_{3}$ & $\mathrm{C}_{64} \mathrm{H}_{80} \mathrm{~N}_{8} \mathrm{O}_{6} \mathrm{~S}_{12}(\mathbf{2}) \cdot 2 \mathrm{CH}_{3} \mathrm{OH}$ \\
Formula weight & $1491 \cdot 36$ & $1474 \cdot 16$ \\
$T(\mathrm{~K})$ & $273(2)$ & $291(2)$ \\
Space group & $P \bar{l}$ & $P \bar{l}$ \\
Crystal system & Triclinic & Triclinic \\
Cell constants & & \\
$a(\AA)$ & $15 \cdot 448(8)$ & $15 \cdot 858(2)$ \\
$b(\AA)$ & $15 \cdot 640(8)$ & $16 \cdot 247(2)$ \\
$c(\AA)$ & $15 \cdot 878(8)$ & $16 \cdot 472(2)$ \\
$\alpha\left(^{\circ}\right)$ & $106 \cdot 702(7)$ & $71 \cdot 279(2)$ \\
$\beta\left(^{\circ}\right)$ & $91 \cdot 659(8)$ & $86 \cdot 046(2)$ \\
$\gamma\left({ }^{\circ}\right)$ & $93 \cdot 213(7)$ & $72 \cdot 668(2)$ \\
Volume $\left(\AA^{3}\right)$ & $3664(3)$ & $3835 \cdot 4(8)$ \\
$Z$ & 2 & 2 \\
Density $\left(\mathrm{g} / \mathrm{cm}^{3}\right)$ & $1 \cdot 352$ & $1 \cdot 276$ \\
Absorption coefficient $\left(\mathrm{mm}^{-1}\right)$ & $0 \cdot 518$ & $0 \cdot 394$ \\
$F(000)$ & 1560 & 1560 \\
Sizes (mm) & $0 \cdot 35 \times 0 \cdot 27 \times 0 \cdot 08$ & $0 \cdot 45 \times 0 \cdot 35 \times 0 \cdot 11$ \\
$\theta\left(^{\circ}\right)$ & $2 \cdot 46$ to $25 \cdot 50$ & $2 \cdot 30$ to $25 \cdot 50$ \\
Limiting indices & $-18 \leq h \leq 18,-18 \leq k \leq 18,-19 \leq 1 \leq 19$ & $19 \leq h \leq 18,-19 \leq k \leq 19,-19 \leq 1 \leq 19$ \\
Reflections collected/unique & $27209 / 13486[R(\mathrm{int})=0 \cdot 1029]$ & $29467 / 14138[R(\mathrm{int})=0 \cdot 0563]$ \\
Data/restraints/parameters & $13486 / 881 / 876$ & $14138 / 537 / 84$ \\
GOF & $0 \cdot 964$ & $1 \cdot 021$ \\
$R_{1}$ & $0 \cdot 1024$ & $0 \cdot 0939$ \\
$W R_{2}$ & $0 \cdot 2421$ & $0 \cdot 2453$ \\
\hline
\end{tabular}

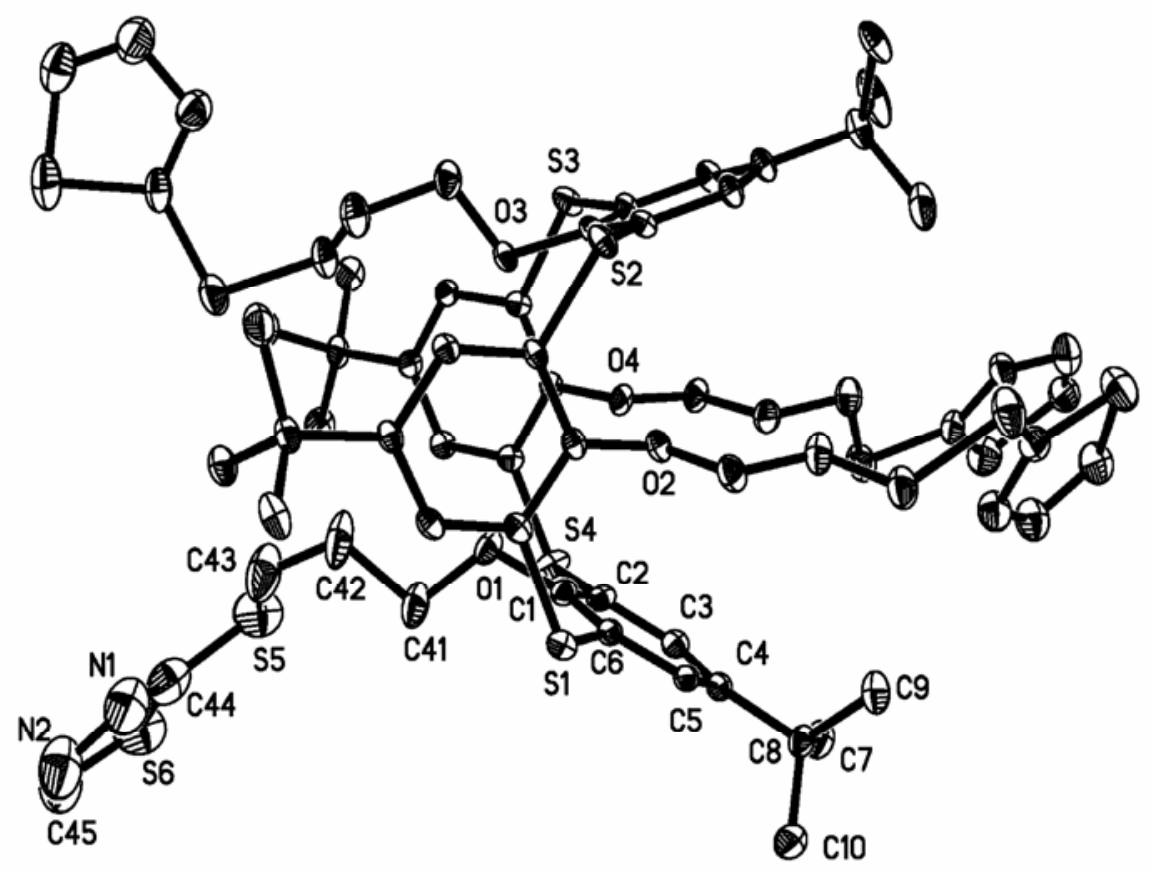

Figure 2. ORTEP of compound 1 with $15 \%$ probability (one chloroform and one water molecules as well as all hydrogen atoms and $t$-butyl groups are deleted for clarity). 
Table 2. Selected bond lengths $(\AA)$ of 1 and 2.

\begin{tabular}{|c|c|c|c|c|c|c|c|}
\hline \multicolumn{4}{|c|}{1} & \multicolumn{4}{|c|}{2} \\
\hline $\mathrm{C}(21)-\mathrm{C}(22)$ & $1 \cdot 390(8)$ & $C(51)-C(52)$ & $1 \cdot 50(1)$ & $\mathrm{C}(21)-\mathrm{C}(22)$ & $1 \cdot 379(8)$ & $\mathrm{S}(3)-\mathrm{C}(31)$ & $1.769(6)$ \\
\hline$C(25)-C(26)$ & $1 \cdot 402(8)$ & $\mathrm{C}(52)-\mathrm{C}(53)$ & $1 \cdot 52(1)$ & $\mathrm{C}(21)-\mathrm{C}(26)$ & $1.402(8)$ & $\mathrm{S}(3)-\mathrm{C}(25)$ & $1.769(6)$ \\
\hline $\mathrm{C}(27)-\mathrm{C}(28)$ & $1 \cdot 50(1)$ & $\mathrm{S}(2)-\mathrm{C}(22)$ & $1.780(6)$ & $C(22)-C(23)$ & $1.400(8)$ & $\mathrm{S}(9)-\mathrm{C}(57)$ & $1.794(9)$ \\
\hline $\mathrm{C}(22)-\mathrm{C}(23)$ & $1 \cdot 395(8)$ & $S(2)-C(16)$ & $1.799(6)$ & $\mathrm{C}(53)-\mathrm{C}(54)$ & $1.488(9)$ & $\mathrm{S}(9)-\mathrm{C}(56)$ & $1.801(9)$ \\
\hline $\mathrm{O}(3)-\mathrm{C}(21)$ & $1 \cdot 384(7)$ & $S(9)-C(54)$ & $1.73(1)$ & $C(54)-C(55)$ & $1 \cdot 58(1)$ & $\mathrm{S}(10)-\mathrm{C}(58)$ & $1.669(8)$ \\
\hline $\mathrm{C}(23)-\mathrm{C}(24)$ & $1 \cdot 387(8)$ & $\mathrm{S}(9)-\mathrm{C}(53)$ & $1.809(8)$ & $C(55)-C(56)$ & $1 \cdot 49(1)$ & $S(10)-C(57)$ & $1.688(8)$ \\
\hline $\mathrm{O}(3)-\mathrm{C}(51)$ & $1 \cdot 414(8)$ & $\mathrm{S}(10)-\mathrm{C}(55)$ & $1 \cdot 66(1)$ & $\mathrm{C}(23)-\mathrm{C}(24)$ & $1 \cdot 350(8)$ & $\mathrm{N}(5)-\mathrm{C}(57)$ & $1 \cdot 289(8)$ \\
\hline $\mathrm{C}(24)-\mathrm{C}(25)$ & $1.405(9)$ & $S(10)-C(54)$ & $1.716(8)$ & $\mathrm{C}(23)-\mathrm{C}(27)$ & $1.551(8)$ & $\mathrm{N}(5)-\mathrm{N}(6)$ & $1 \cdot 397(8)$ \\
\hline $\mathrm{C}(24)-\mathrm{C}(27)$ & $1 \cdot 561(9)$ & $\mathrm{N}(5)-\mathrm{C}(54)$ & $1 \cdot 30(1)$ & $C(24)-C(25)$ & $1.401(8)$ & $\mathrm{N}(6)-\mathrm{C}(58)$ & $1 \cdot 260(9)$ \\
\hline $\mathrm{N}(5)-\mathrm{N}(6)$ & $1 \cdot 34(1)$ & $\mathrm{N}(6)-\mathrm{C}(55)$ & $1 \cdot 26(1)$ & $C(25)-C(26)$ & $1 \cdot 393(8)$ & $\mathrm{C}(27)-\mathrm{C}(28)$ & $1 \cdot 47(1)$ \\
\hline
\end{tabular}

Table 3. Selected bond angles (deg) of 1 and 2.

\begin{tabular}{lllll}
\hline \multicolumn{2}{c}{1} & & \multicolumn{2}{c}{2} \\
\cline { 1 - 2 } $\mathrm{C}(23)-\mathrm{C}(22)-\mathrm{S}(2)$ & $115 \cdot 5(4)$ & & $\mathrm{C}(26)-\mathrm{C}(21)-\mathrm{S}(2)$ & $122 \cdot 9(4)$ \\
$\mathrm{C}(26)-\mathrm{C}(21)-\mathrm{O}(3)$ & $120 \cdot 2(6)$ & & $\mathrm{O}(3)-\mathrm{C}(26)-\mathrm{C}(25)$ & $121 \cdot 0(5)$ \\
$\mathrm{C}(21)-\mathrm{C}(26)-\mathrm{S}(3)$ & $122 \cdot 7(5)$ & & $\mathrm{O}(3)-\mathrm{C}(53)-\mathrm{C}(54)$ & $107 \cdot 8(6)$ \\
$\mathrm{O}(3)-\mathrm{C}(51)-\mathrm{C}(52)$ & $110 \cdot 2(7)$ & & $\mathrm{C}(53)-\mathrm{C}(54)-\mathrm{C}(55)$ & $111 \cdot 6(6)$ \\
$\mathrm{C}(51)-\mathrm{C}(52)-\mathrm{C}(53)$ & $112 \cdot 2(6)$ & & $\mathrm{C}(56)-\mathrm{C}(55)-\mathrm{C}(54)$ & $109 \cdot 4(7)$ \\
$\mathrm{C}(52)-\mathrm{C}(53)-\mathrm{S}(9)$ & $112 \cdot 6(6)$ & & $\mathrm{C}(55)-\mathrm{C}(56)-\mathrm{S}(9)$ & $110 \cdot 7(7)$ \\
$\mathrm{N}(5)-\mathrm{C}(54)-\mathrm{S}(9)$ & $125 \cdot 1(7)$ & & $\mathrm{S}(10)-\mathrm{C}(57)-\mathrm{S}(9)$ & $126 \cdot 5(5)$ \\
$\mathrm{S}(10)-\mathrm{C}(54)-\mathrm{S}(9)$ & $122 \cdot 5(6)$ & & $\mathrm{N}(5)-\mathrm{C}(57)-\mathrm{S}(9)$ & $120 \cdot 0(7)$ \\
$\mathrm{C}(22)-\mathrm{S}(2)-\mathrm{C}(16)$ & $106 \cdot 8(3)$ & & $\mathrm{C}(26)-\mathrm{O}(3)-\mathrm{C}(53)$ & $115 \cdot 0(4)$ \\
$\mathrm{C}(26)-\mathrm{S}(3)-\mathrm{C}(32)$ & $109 \cdot 6(3)$ & & $\mathrm{C}(57)-\mathrm{S}(9)-\mathrm{C}(56)$ & $98 \cdot 0(4)$ \\
$\mathrm{C}(54)-\mathrm{S}(9)-\mathrm{C}(53)$ & $101 \cdot 9(4)$ & & $\mathrm{C}(15)-\mathrm{S}(2)-\mathrm{C}(21)$ & $108 \cdot 5(3)$ \\
$\mathrm{C}(21)-\mathrm{O}(3)-\mathrm{C}(51)$ & $113 \cdot 0(5)$ & & $\mathrm{C}(31)-\mathrm{S}(3)-\mathrm{C}(25)$ & $107 \cdot 7(3)$ \\
\hline
\end{tabular}

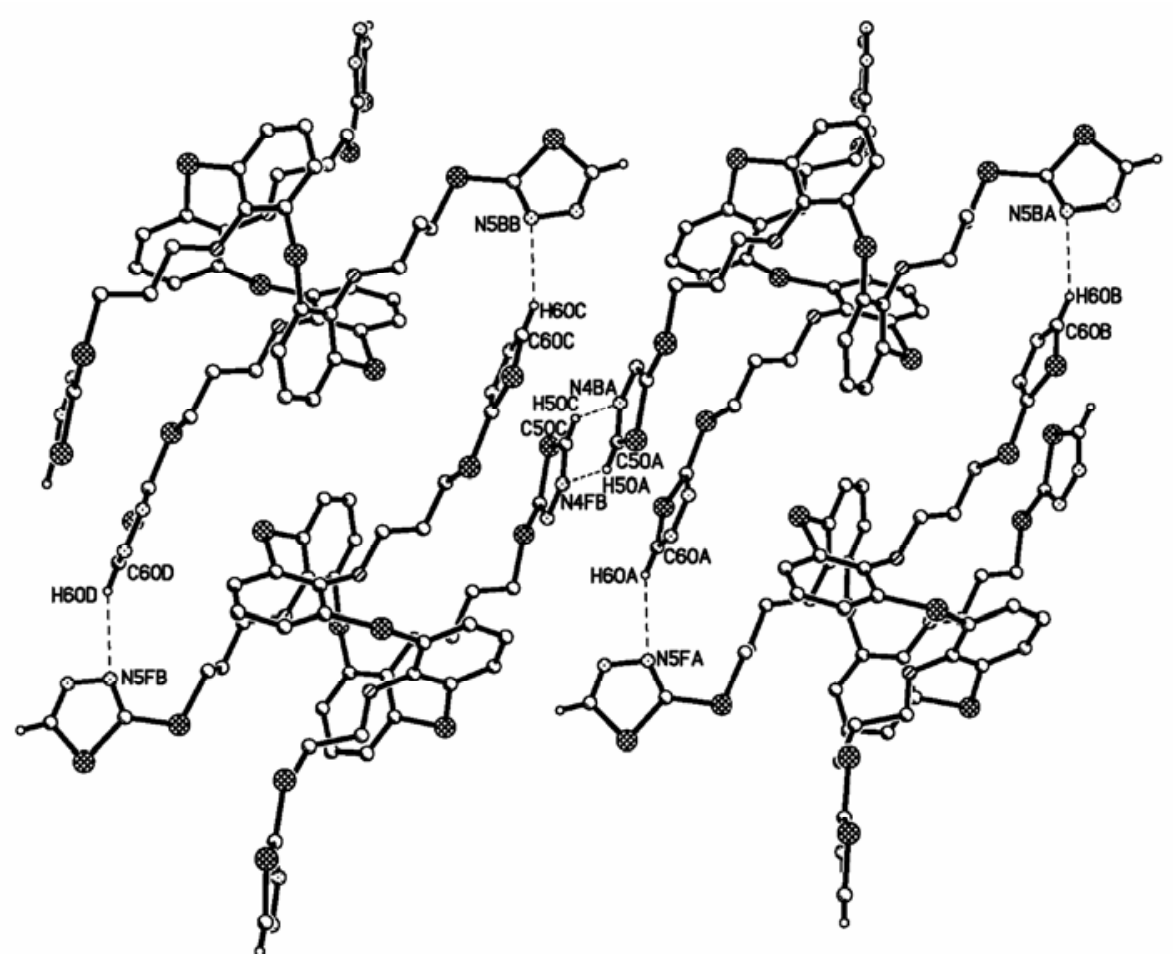

Figure 3. 1-D chain of compound 1 formed by $\mathrm{C}-\mathrm{H} \cdots \mathrm{N}$ interactions (chloroform and water molecules as well as all $t$-butyl groups are deleted and only the hydrogen atoms involved in hydrogen bonding are shown for clarity). 
Table 4. Geometrical parameters of hydrogen bonds for 1 and 2 ( $\AA$, deg).

\begin{tabular}{|c|c|c|c|c|c|}
\hline Compounds & $\mathrm{D}-\mathrm{H} \cdots \mathrm{A}$ & $d(\mathrm{D}-\mathrm{H})$ & $d(\mathrm{H} \cdots \mathrm{A})$ & $d(\mathrm{D} \cdots \mathrm{A})$ & $\angle(\mathrm{D}-\mathrm{H} \cdots \mathrm{A})$ \\
\hline \multirow[t]{4}{*}{1} & 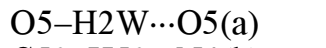 & $0 \cdot 96$ & 1.78 & $2 \cdot 28(3)$ & $108 \cdot 0$ \\
\hline & 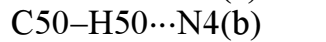 & 0.93 & $2 \cdot 54$ & $3 \cdot 25(1)$ & $147 \cdot 0$ \\
\hline & C60-H60 ‥N5(c) & 0.93 & $2 \cdot 55$ & $3 \cdot 40(2)$ & $153 \cdot 0$ \\
\hline & O5B-H1B $\cdots$ S12A(d) & 0.962 & $2 \cdot 903$ & $3 \cdot 78(7)$ & $179 \cdot 7$ \\
\hline \multirow[t]{7}{*}{2} & $\mathrm{O} 5-\mathrm{H} 5 \cdots \mathrm{O} 6$ & $0 \cdot 82$ & $2 \cdot 33$ & $3 \cdot 05(5)$ & $146 \cdot 0$ \\
\hline & 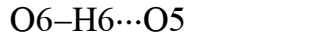 & $0 \cdot 82$ & $2 \cdot 45$ & $3 \cdot 05(5)$ & $130 \cdot 0$ \\
\hline & C46-H46‥O6(e) & $0 \cdot 93$ & $2 \cdot 55$ & $3 \cdot 42(3)$ & $156 \cdot 0$ \\
\hline & C65-H65A $\cdots \mathrm{O} 6$ & $0 \cdot 96$ & $2 \cdot 45$ & $3 \cdot 14(7)$ & $129 \cdot 0$ \\
\hline & C65-H65B ‥N2(f) & 0.95 & $2 \cdot 43$ & $3 \cdot 35(5)$ & $162 \cdot 0$ \\
\hline & C49B-H49D $\cdots \mathrm{S} 2 \mathrm{~A}$ & 0.97 & $2 \cdot 93$ & $3 \cdot 70$ & $147 \cdot 2$ \\
\hline & C49A-H49B $\cdots$ S2B & 0.97 & $2 \cdot 93$ & $3 \cdot 70$ & $147 \cdot 2$ \\
\hline
\end{tabular}

Symmetry codes: a: $1-x,-y,-z ; \mathrm{b}: 2-x, 1-y, 2-z ; \mathrm{c}: 2-x, 1-y, 1-z ; \mathrm{d}:-x+2$, $-y+1,-z+1 ; \mathrm{e}: x, 1+y,-1+z ; \mathrm{f}: 1-x, 1-y,-z$.

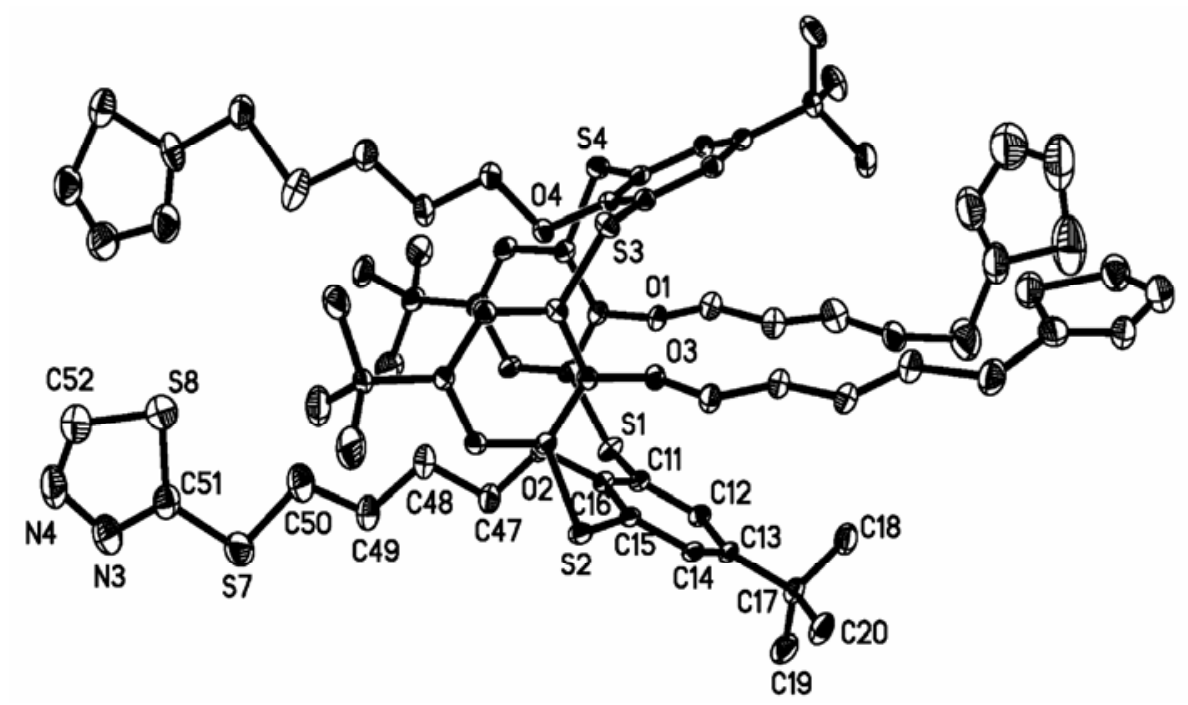

Figure 4. ORTEP of compound 2 with $15 \%$ probability (two methanol molecules and all hydrogen atoms are omitted for clarity).

interactions, ${ }^{16,17}$ which act as bridges to link the 1-D chains and methanol dimers (see supplementary material figure S5).

\section{Conclusion}

In summary, crystal structures of two thiacalix[4] arene derivatives anchoring thiadiazole functional groups at lower rims, $\mathrm{C}_{60} \mathrm{H}_{72} \mathrm{O}_{4} \mathrm{~S}_{12} \mathrm{~N}_{8}$ (1), $\mathrm{C}_{64} \mathrm{H}_{80} \mathrm{O}_{4}$ $\mathrm{S}_{12} \mathrm{~N}_{8}$ (2), have been determined by single-crystal $\mathrm{X}$-ray diffraction. The thiacalix[4]arene platforms in both compounds 1 and 2 adopt simple 1,3-alternate conformation. This may be ascribed to the introduction of a long chain, which prevents the rotation of the aryl rings in thiacalixarene skeleton. Both structures of $\mathrm{C}_{60} \mathrm{H}_{72} \mathrm{O}_{4} \mathrm{~S}_{12} \mathrm{~N}_{8}$ (1). $\mathrm{CHCl}_{3} \cdot \mathrm{H}_{2} \mathrm{O}$ and
$\mathrm{C}_{64} \mathrm{H}_{80} \mathrm{~N}_{8} \mathrm{O}_{6} \mathrm{~S}_{12}$ (2).2 $\mathrm{CH}_{3} \mathrm{OH}$ display 3-D and 2-D supramolecular network respectively through intermolecular interactions.

\section{Acknowledgements}

This work was supported by the Natural Science Foundation of China (No. 20872058).

\section{Supplementary materials}

Crystallographic data for 1 (CCDC-642372) and 2 (CCDC-642374) reported in this paper have been deposited with the Cambridge Crystallographic Data Centre as supplementary materials. Copies of this 
material can be obtained free of charge from Director, CCDC. 12 Union Road, Cambridge CB2 1EZ, UK (Fax: +44-1223-336033; e-mail: deposit@ccdc.cam.ac.uk).

\section{References}

1. Gutsche C D 1989 Monographs in supramolecular chemistry, calixarenes (ed.) J F Stoddart (Cambridge: The Royal Society of Chemistry)

2. Gutsche C D 1998 Calixarenes revisited (Cambridge: The Royal Society of Chemistry)

3. Mandolini L and Ungaro R 2000 Calixarenes in action (London: Imperial College Press)

4. Asfari Z, Böhmer V, Harrowfield J M and Vicens J 2001 Calixarenes 2001 (Dordrecht: Kluwer Academic Publishers)

5. Kumagai H, Hasegawa M, Miyanari S, Sugawa $Y$, Sato Y, Hori T, Ueda S, Kaniyama $\mathrm{H}$ and Miyano $\mathrm{S}$ 1997 Tetrahedron Lett. 383971

6. Lhoták P 2004 Eur. J. Org. Chem. 1675
7. Morohashi N, Narumi F, Iki N, Hattori T and Miyano S 2006 Chem. Rev. 1065291

8. Atwood J L, Barbour L J and Jerga A 2002 Chem. Commun. 2952

9. Lhoták P, Himl M, Stibor I, Sýkora J, Dvořáková H, Lang J and Petříčková H 2003 Tetrahedron 597581

10. Zhao B T, Zhou Z and Yan Z N 2007 Heterocycles 712211

11. Sheldrick G M 1997 SHELXS-97 Program for solution of crystal structures University of Göttingen, Germany 1990

12. Bruker 1997 SMART, SAINT and SHELXTL (Madison, Wisconsin, USA: Bruker AXS Inc.)

13. Sheldrick G M 1997 SHELXL-97 Program for refinement of crystal structures University of Göttingen, Germany

14. Wang L, Zhang J, Ye B X, Hu P Z and Zhao B T 2006 Acta Crystallogr. E62, 04844

15. Andreu R, Malfant I, Lacroix P G and Cassonx P 2000 Eur. J. Org. Chem. 737

16. Jeffery G A 2003 Crystallogr. Rev. 9135

17. Steiner T 2003 Crystallogr. Rev. 9177

18. Jia C, Liu S X, Ambrus C, Neels A, Labat G and Decurtins S 2006 Inorg. Chem. 453152 